\title{
La sociedad y la comunicación desde la perspectiva de Manuel Castells de sociedad red
}

\section{Society and communication from the perspective of Manuel Castells network society}

José Luis Flores Torres*

Universidad Multicultural Emilio Cárdenas (CUDEC)

Atlacomulco número 191 y 193, La Loma,

C. P. 54060, Tlalnepantla, Estado de México

Editor: Rogelio del Prado Flores

Fecha de recepción: 29 de marzo 2020

Fecha de aceptación: 9 de mayo 2020 jose.floresto@anahuac.mx

https://orcid.org/0000-0001-911-5812

https://doi.org/10.36105/stx.2020n5.05

\section{RESUMEN}

La sociedad red es una manera de entender el mundo propuesta por Manuel Castells desde hace ya más de dos décadas. A partir de dicho concepto el autor español explica cómo es que el advenimiento de las tecnologías digitales generó cambios tan profundos que impactaron no solo en los procesos de producción, sino en la manera en la que las personas viven su cotidianidad. Es decir, de acuerdo con Castells, en este nuevo contexto (2009, p. 154) los individuos están reconstruyendo el modelo de interacción social con la ayuda de las nuevas posibilidades tecnológicas para crear un nuevo modelo de sociedad: la sociedad red.

Así, los conceptos sociedad y comunicación cobran un nuevo sentido en dicho paradigma. Por ello, el presente artículo analizará tales acepciones subrayando la manera en la que Castells las concibe y las relaciona a su vez con otros conceptos (como la libertad y la tecnología). De igual manera, tales conceptos (sociedad y comunicación) serán contrastados con la visión que aportan Byung-Chul Han (con el cual se contrastará el concepto de sociedad) y Marshall McLuhan (con quien se contrastará el concepto comunicación).

\footnotetext{
* Doctorante en Investigación de la Comunicación por la Universidad Anáhuac México. Maestro en Comunicación por la Universidad Iberoamericana en donde se tituló con Mención honorífica. Licenciado en Ciencias de la Comunicación por la Universidad Autónoma Metropolitana (Xochimilco). Docente en la Facultad de Comunicación de la Universidad Anáhuac México desde hace io años. Ha impartido cursos y ponencias a nivel nacional e internacional en países como Ecuador y España.
} 
De tal manera que el análisis aquí propuesto buscará contribuir a entender las amplias implicaciones contenidas en el concepto sociedad red no solo a partir de la visión de Manuel Castells, sino además buscando la comparación y el contraste con otras visiones que de los mismos conceptos poseen otros autores, en este caso Han y McLuhan.

Palabras clave: Sociedad red, internet, comunicación, tecnología.

\section{ABSTRACT}

The Network Society is a way of understanding the world proposed by Manuel Castells for more than two decades. Based on this concept, the spanish author explains how the advent of digital technologies generated changes so profound that they impacted not only on production processes but on the way people live their daily lives. That is, according to Castells in this new context (2009, p. 154), individuals are rebuilding the model of social interaction with the help of new technological possibilities to create a new model of society: the network society.

Thus, the concepts of society and communication take on a new meaning in this paradigm. For this reason, this article will analyze short stories, highlighting the way in which Castells conceives them and in turn relates them to other concepts (such as freedom and technology). Similarly, story concepts (society and communication) will be contrasted with the vision provided by Byung-Chul Han (with which the concept of society will be contrasted) and Marshall McLuhan (with whom the concept of communication will be contrasted).

In this way, the analysis proposed here will seek to contribute to understanding the broad implications contained in the concept of red society, not only from the vision of Manuel Castells, but also seeking comparison and contrast with other visions of the same concepts as other authors, in this case Han and McLuhan.

Keywords: Network Society, Internet, communication, tecnology.

\section{INTRODUCCIÓN}

$\mathrm{E}^{\mathrm{s}}$ ser humano, desde épocas primitivas, se dio cuenta tanto de lo fundamental que resultaba ser gregario como de la importancia del trabajo en grupo para obtener logros que aseguraran la supervivencia de la colectividad generando poco a poco formas de organización para el trabajo, modelos de estructura social y formas de comunicación cada vez 
más complejas. Es decir, la vida en sociedad en mayor o menor medida siempre se ha caracterizado por involucrar invariablemente un ir y venir de pequeñas y grandes revoluciones (en todos los órdenes) que no se detienen y que han permeado la presencia del ser humano en la tierra.

Así desde la década de los años sesenta del siglo pasado el hombre empezó a situarse en los albores de una era que ha sido llamada de diferentes formas: modernidad, postmodernidad, era de la información, globalización, sociedad red, etc. Dicho momento, a su vez, sustituyó otro entorno que había sido profundamente revolucionario y que fue llamado sociedad industrial, que había alcanzado su madurez a partir del siglo XVIII y que vio nacer a los medios de comunicación masiva a finales del siglo XIX.

Este tránsito de la sociedad moderna a la digital tal parece que no ha concluido del todo, ya que mucho de ese mundo industrial analógico sigue presente en los haceres cotidianos de la gente. No obstante, desde los años sesenta del siglo XX se empezaron a gestar una serie de cambios no solo en el ámbito de la tecnología sino, como lo señala Manuel Castells (200I, p. I9), surgió un nuevo modelo socio-técnico.

Este entorno produjo a su vez cambios que no han sido solo tecnológicos, sino que han significado auténticas revoluciones sociales, culturales y, desde luego, económicas que han impactado en los procesos de producción, distribución y consumo de productos. $\mathrm{O}$ bien, como lo señalan Terceiro y Matías (200I, p. 5I), la estructura que caracteriza a la sociedad digital se deriva de una serie de cambios sociales y económicos. Esta nueva sociedad ha alterado el tipo de relaciones humanas cuantitativa y cualitativamente.

En muchos sentidos este contexto lleno de transformaciones fue generado por el nacimiento y evolución de la primera generación de Internet, que según Ronda Hauben (I993) fue el resultado de la colaboración científica y técnica internacional que permitió que los primeros proyectos concretos de redes de computadoras se empezaran a desarrollar en la zona de Silicon Valley, California.

De esta manera la revolución tecnológica que había surgido en el contexto de la guerra fría dio como resultado en 1969 el nacimiento de las primeras redes de computadoras en el seno del proyecto ARPANET (en el entorno de la comunidad académica y militar estadounidense) que estuvieron en capacidad de compartir archivos con información entre universidades e institutos tecnológicos en tiempo real. Era el inicio de lo que poco más de dos décadas más tarde se conoció comercialmente como Internet.

Con el arribo de esta tecnología se generaron cambios suficientemente profundos para afirmar que el ser humano estaba empezando a transitar del paradigma industrial, a lo que se empezó a llamar sociedad de la información o como lo detalla Manuel Castells, el modelo de sociedad red que tiene su originalidad en el hecho de que se constituye no solo como 
tecnológico sino al mismo tiempo sociocultural en donde, subraya el propio autor (2006, p. 32), se establece una sociedad constituida alrededor de las tecnologías de la información basadas en la microelectrónica.

Para tener una idea de la manera en la que la cultura digital ha impactado en la vida social podemos señalar el caso de México, en donde datos del $15^{\circ}$ Estudio sobre los Hábitos de los usuarios de Internet en México, publicado por la Asociación de Internet Mx (2019), señalan que, al segundo trimestre de 2019, 71\% de la población de seis años o más en México se declaró usuaria de Internet (lo cual equivale a 79.I millones de mexicanos). Es decir que se debe reconocer que la presencia de la tecnología digital está en plena expansión no solo en el entorno personal, sino también en el laboral, escolar, comercial, etc. No obstante, también es justo señalar que de acuerdo con las cifras antes referidas podemos observar que casi $30 \%$ de los mexicanos están formalmente marginados del aprovechamiento de esta plataforma. Tal porcentaje representa asimismo a personas que no solamente han sido marginadas de la cultura digital, sino que tal rechazo es consecuencia de décadas de marginación social, cultural, laboral y económica.

Por tales razones el paso del paradigma industrial al de la sociedad red de alguna manera ha generado que diversos autores desde diferentes perspectivas se hallan dado a la tarea no solamente de describir y analizar tal transición, sino que además empezaran a generar una perspectiva crítica de este nuevo modelo de organización social. Así, en el centro de la presente reflexión se situará como objeto de discusión el concepto sociedad red, propuesto por Manuel Castells para caracterizar la lógica del mundo actual marcada por una suerte de cultura emanada de Internet.

Dicha denominación será analizada en el presente artículo a partir de dos elementos que la configuran: el concepto de sociedad y el de comunicación, los cuales empezaron a cobrar nuevas dimensiones a partir de la emergencia de la cultura digital. Tales conceptos serán analizados no solo desde la visión de Manuel Castells, sino que se buscará contrastarlo con la perspectiva que de los mismos plantean Byung-Chul Han y Marshal McLuhan buscando tener una perspectiva más amplia de tales conceptos y a su vez permita una plena comprensión de la llamada por Castells sociedad red.

\section{MARCO TEÓRICO}

Uno de los autores más emblemáticos de la cultura digital es el pensador español Manuel Castells Oliván, quien desde la perspectiva de la sociología urbanista percibe en primera instancia que la manera en la que conviven lo social, el hombre contemporáneo y la tecno- 
logía se asemeja a la estructura de una red que depende para su funcionamiento de algo que llama conectividad.

Para entender la lógica de este modelo de sociedad habría que aclarar en primera instancia que para Castells (2006, p. 28) una red es un conjunto de nodos interconectados. Un nodo, a su vez, es el punto de intersección de una curva. Esto es: puntos de contacto humano y al mismo tiempo ocasión para la generación de diversas formas de interacción comunicativa. Además, a diferencia de los modelos de organización social en red tradicionales, las redes emanadas de la tecnología digital no poseen ningún centro, solo nodos.

Es decir, para Castells (2006, p. 58) las redes (tradicionales) eran una extensión del poder centrado en lo alto de las organizaciones verticales que configuraron la historia de la humanidad. En cambio, en las redes emanadas de la cultura digital el poder se encuentra por así decirlo descentralizado y los nodos (es decir cualquier usuario de la red) pueden tener mayor o menor importancia cuando absorben más información relevante y la procesan más eficientemente.

De acuerdo con esto cualquier ser humano podría estar en condiciones de convertirse en ciudadano copartícipe de la cultura de la libertad y la participación propia de una cultura digital que promete enlazar a todos con el mundo. No obstante, en la realidad la conectividad se constituye como el factor que a la vez que enlaza a algunos, margina a otros.

Así el individuo en la sociedad red es nodo que existe en tanto pueda vincularse con otras personas a través de la pantalla. Pero estos vínculos en la red en realidad pocas veces llegan a ser tan profundos y duraderos como los que existen en el mundo offline. Es decir, en Internet el ser humano es nodo/ciudadano/prosumer inevitablemente acoplado a la tecnología y en el mejor de los escenarios copartícipe de este nuevo modelo de sociedad.

Otra diferencia entre las redes de organización tradicionales y las propias dela sociedad red de acuerdo con Manuel Castells (2006, p. 27), consiste en que en el entorno contemporáneo la sociedad red es una estructura social que está compuesta por redes potenciadas de tecnologías de la información y de la comunicación basadas en la microelectrónica. Se trata pues de un conjunto de entramados que dependen para su funcionamiento de la tecnología digital la cual no solamente condiciona su existencia, sino que por sus características potencializa su alcance.

Ante esto, el propio Castells señala tres características fundamentales y diferenciadores de la sociedad red (2006, p. 34): Su capacidad autoexpansiva de procesamiento y de comunicación en términos de volumen, complejidad y velocidad; su capacidad para recombinar basada en la digitalización y en la comunicación recurrente, y su flexibilidad de distribución mediante redes interactivas y digitalizadas.

Es así como la no existencia de centros resulta ser una de las principales características de la sociedad red en donde todos los participantes son nodos que, de acuerdo con sus capacidades e intereses, expanden o potencializan sus formas tanto de informar como de 
atraer información, además de que se recombinan de acuerdo con intereses particulares y/o contextuales y flexibilizan su distribución en relación con los intereses de los usuarios con quienes se suele interactuar.

De tal manera, para Manuel Castells la comunicación en la sociedad red está forzosamente ligada a la tecnología. Para tal efecto, el propio autor concibe a la comunicación en el entorno de la sociedad red como (2006, p. 88) interactiva caracterizada por la capacidad para enviar mensajes a muchas personas, en tiempo real, o en un momento concreto, y con la posibilidad de usar la comunicación puntoapunto, estando el alcance de su difusión en función de las características de la práctica comunicativa perseguida.

Así, esta perspectiva de sociedad red está muy relacionada con un modelo de comunicación (también planteado por Castells) que pasa del modelo de comunicación circular propio de la comunicación interpersonal (rica en niveles y posibilidades de retroalimentación directa) y de la comunicación lineal planteada por la Mass Communication Research. Castells en su propuesta observa un proceso de comunicación multinivel (que incluye lo interpersonal, lo grupal y lo global), reticular, participativo, con formas de retroalimentación diversas y que se adapta a las necesidades de los diferentes públicos.

\section{METODOLOGÍA}

El presente texto representa una aproximación al mundo digital y en específico a las implicaciones del concepto sociedad red visto desde la perspectiva del sociólogo urbanista español Manuel Castells. Tal reflexión forma parte del marco teórico-conceptual de la tesis doctoral en construcción en la que se analizan las formas de interacción que los migrantes venezolanos avecindados en México construyen en sus comunidades virtuales y utilizan para establecer contacto y generar redes de apoyo y solidaridad. Dadas las características del tema, el concepto sociedad red resulta fundamental para entender la manera en la que este grupo migrante experimenta ser parte de la diáspora venezolana recurriendo a las herramientas digitales para generar formas de interacción digital que se traducen en la constitución de acciones tendientes a mejorar sus condiciones de vida en México.

En tal sentido comprender plenamente la lógica de la sociedad red representa un imperativo para dar sentido a la manera en la que esta comunidad migrante accede a estas plataformas y de alguna manera vive parte de esta diáspora vinculándose a las comunidades que se generan en el espacio virtual.

Por ello, en el presente artículo se utilizó como método el análisis de contenido comparativo que tendrá como tarea en primera instancia establecer conceptos clave implícitos en 
la definición de sociedad red. Así, tal como lo señala Alva (200I, p.3) una etapa esencial del estudio lo representa el desglose del mensaje en unidades materiales discretas, clasificadas en categorías.

En tal caso los conceptos o categorías a analizar son precisamente sociedad y comunicación. Tales conceptos clave habrán de constituir para el presente estudio unidades de análisis las cuales habrán de ser analizadas y contrastadas con la perspectiva que tienen de tales conceptos Byung-Chul Han (sociedad) y Marshall McLuhan (comunicación). Dichos conceptos, a su vez, serán vinculados con otros que de ahí se desprenden, como es el caso del sentido de la libertad y la tecnología.

De esta manera el presente documento tiene como objetivo analizar las implicaciones del concepto sociedad red a partir de los conceptos sociedad y comunicación examinándolos tanto desde la óptica de Manuel Castells, como de Byung-Chul Han y Marshall McLuhan respectivamente. Esto habrá de mostrar una perspectiva amplia del fenómeno sociedad red al desglosarlo y analizarlo a partir de algunos de sus componentes conceptuales fundamentales.

Así resulta fundamental entender en primera instancia que como antecedente de los análisis de contenido Alva (200I, p.3) señala que en sus inicios pretendía ser una técnica investigativa para la descripción objetiva, sistemática y cuantitativa del contenido manifiesto de la comunicación. Berelson, por su parte (1984, p. I8), define el análisis de contenido como una técnica de investigación para la descripción objetiva y sistemática del contenido manifiesto de las comunicaciones de cualquier tipo.

Cabe señalar que los conceptos sociedad y comunicación fueron elegidos para ser analizados en el presente estudio dado que en gran medida el concepto sociedad red descansa en tales acepciones alrededor de la cual giran otros conceptos que también serán abordados como el sentido de la libertad y la tecnología.

Así para el desarrollo del análisis de contenido, en primera instancia se habrán de citar los conceptos sociedad y comunicación desde las perspectivas señaladas. Después se llevará a cabo un análisis de cada concepto a partir de las ideas plasmadas por los autores en sus textos. Posteriormente, se elaborará una tabla en donde se analizarán coincidencias y diferencias que existen entre los enfoques señalados. De igual manera se elaborará una tabla de valoración del concepto para analizar el sentido (positivo o negativo) que poseen los conceptos analizados, esto a partir de lo que señalan los propios autores, de las palabras que utilizan o los conceptos con los que los relacionan. Por último, se desarrollará una conclusión que dé cuenta del resultado del análisis comparativo entre tales perspectivas. 
DESARROLLO

\section{Concepto de sociedad}

\begin{tabular}{|l|l|}
\hline \multicolumn{1}{|c|}{ PERSPECTIVA DE MANUEL CASTELLS } & \multicolumn{1}{|c|}{ PERSPECTIVA DE BYUNG-CHUL HAN } \\
\hline $\begin{array}{l}\text { Para Castells implica (2006, p. 27) todos aquellos } \\
\text { acuerdos organizativos humanos en relación con } \\
\text { la producción, el consumo, la reproducción, la } \\
\text { experiencia y el poder, expresados mediante una } \\
\text { comunicación significativa codificada por la cultura. }\end{array}$ & $\begin{array}{l}\text { Byung-Chul Han (2002, p. 45) observa a la } \\
\text { sociedad como colectividades agotadas por los } \\
\text { excesos y en vías de convertirse en una sociedad } \\
\text { de dopaje. }\end{array}$ \\
\hline
\end{tabular}

FUENTE: ELABORACIÓN PROPIA.

Para Manuel Castells, el concepto sociedad red resulta fundamental para entender la lógica de la vida contemporánea. Así, al conceptualizar a la sociedad, dicho autor señala que implica (2006, p. 27) todos aquellos acuerdos organizativos humanos en relación con la producción, el consumo, la reproducción, la experiencia y el poder, expresados mediante una comunicación significativa codificada por la cultura.

Es decir, Castells concibe al conglomerado social como un ente ampliamente vinculado, con redes de contactos, capaz de generar acuerdos y que tiene como columna vertebral la tecnología la cual resulta detonante para generar nuevas sensaciones, formas de consumo, relaciones para el trabajo y en suma una cultura vinculada a la tecnología digital.

Tales avances tecnológicos permiten que la relaciones y contactos en la sociedad red sean globales. La sociedad red, señala Castells (2009, p. 5I) es pues una sociedad global. Así, lo paradójico del caso es que, a pesar del alcance global de tales redes de relación no todos pueden o quieren formar parte de ella, no obstante, todo el mundo se ve afectado por este nuevo modelo de sociedad.

Así, Internet sería la base significativa a partir de la cual la sociedad red se diferenciaría de otros modelos de sociedad, no solo por la posibilidad que provee para generar contactos comunicativos, sino por la eficiencia y rapidez que le estaría aportando al proceso de difusión de los mensajes gracias a la tecnología digital.

Aquí, vale la pena observar la importancia que Castells le proporciona no solo a la comunicación, sino a la información como parte central en la constitución de la sociedad red. Dicha importancia tal parece que no solo radica en la cantidad de datos que cada nodo (o ciudadano conectado a la red) absorbe y/o procesa dentro de la sociedad red, sino de la relevancia de dicha información para el cumplimiento de sus objetivos. 
De igual manera, Manuel Castells vincula su modelo de sociedad al concepto de libertad, ya que a diferencia de las redes tradicionales, en donde su funcionamiento se vinculaba a estructuras de poder centralizadas con roles bien establecidos, la sociedad red emerge de una cultura de la libertad y la participación amplia.

Por su parte, Byung-Chul Han coincide en entender a las sociedades como ampliamente vinculadas a la tecnología emanada de Internet. No obstante, advierte que a las sociedades actuales se les puede agregar la denominación sociedades del rendimiento. De esta manera, Han no percibe a las sociedades contemporáneas vinculadas a la información o al conocimiento, sino que las entiende a partir de su cansancio, es decir, (2002, p. 45) observa a la sociedad como colectividades agotadas por los excesos. Así, estaría refiriéndose a una sociedad activa, pero cansada y en vías de convertirse (2002, p. 45) en una sociedad de dopaje.

Tal cansancio representaría para para Han una sensación particular en una sociedad vinculada a las redes globales, pero que vive su cansancio, como señala el autor (20I2, p. 46), a solas. Es el cansancio del hombre abrumado, fundido por las autoexigencias propias del sistema.

Así, en Han distinguimos que las tecnologías digitales, más que formar parte de una cultura de la libertad y de la participación (como se establece en Castells), estarían formando parte fundamental del engranaje del sistema neoliberal el cual concibe como un sistema muy eficiente, incluso inteligente para explotar la libertad. Se explota todo aquello que pertenece a prácticas y formas de libertad como la emoción, el juego y la comunicación, señala Han (20I4, p. 8).

De hecho, en el fondo lo que critica Han no es la cultura de la libertad emanada de Internet, sino que esta es mera ilusión. Y no solo eso, sino que esa supuesta libertad lleva implícita su contraparte, pues es en realidad una gustosa atadura (que divierte y al mismo tiempo dopa) ya que, según señala el autor (2014, p. II), la libertad y la comunicación ilimitadas se convierten en control y vigilancia totales. También los medios sociales se equiparan cada vez más a panópticos digitales que vigilan y explotan lo social de forma despiadada.

\section{COINCIDENCIAS/DIFERENCIAS ENTRE CASTELLS Y HAN}

COINCIDENCIAS

Ambas perspectivas coinciden en que las sociedades actuales están fuertemente influidas por las tecnologías digitales.

\section{DIFERENCIAS}

Desde la perspectiva de Castells, la sociedad red se caracterizó por diferenciarse de los antiguos modelos de sociedad al proponer un modelo de red participativa en donde el poder estaría descentralizado y en donde todos se pueden influir mutuamente. 


\begin{tabular}{|l|l|}
\hline \multicolumn{1}{|c|}{ COINCIDENCIAS } & \multicolumn{1}{c|}{ DIFERENCIAS } \\
\hline $\begin{array}{l}\text { En ambas se relaciona el concepto } \\
\text { sociedad con el sentido de la libertad. }\end{array}$ & $\begin{array}{l}\text { Para Han, las sociedades actuales son conglomerados agotados } \\
\text { por la autoexigencia y la excesiva vigilancia autoinfligida. }\end{array}$ \\
\hline & $\begin{array}{l}\text { Para Castells, la sociedad red se caracteriza por surgir de una } \\
\text { cultura de la libertad y la partipación la cual fomenta y valora. }\end{array}$ \\
\hline & $\begin{array}{l}\text { Para Han,la libertad en las sociedades contemporáneas no solo } \\
\text { es ficticia o artificial, sino que en realidad esta forma de libertad } \\
\text { significa su contrario, es decir atadura a un sistema neoliberal } \\
\text { que lo explota sin que se percate de esto. }\end{array}$ \\
\hline
\end{tabular}

FUENTE: ELABORACIÓN PROPIA.

\section{VALORACIÓN DE LOS CONCEPTOS}

\begin{tabular}{|l|l|l|}
\hline \multicolumn{1}{|c|}{ CONCEPTO } & \multicolumn{1}{|c|}{ MANUEL CASTELLS } & \multicolumn{1}{c|}{ BYUNG-CHUL HAN } \\
\hline Sociedad & $\begin{array}{l}\text { Para Manuel Castells el concepto de } \\
\text { sociedad (y en particular de sociedad } \\
\text { red) adquiere un sentido positivo (aun } \\
\text { cuando reconoce que hay ciudadanos } \\
\text { que son marginados de sus beneficios), } \\
\text { ya que se encuentran vinculadas en } \\
\text { redes potenciadas por la tecnología que } \\
\text { les permite vincularse a un nivel global. }\end{array}$ & $\begin{array}{l}\text { Se percibe una connotación negativa } \\
\text { en la perspectiva que sobre el concepto } \\
\text { sociedad desarrolla Han, ya que vincula } \\
\text { a las sociedades contemporáneas con } \\
\text { los conceptos cansancio, rendimiento, } \\
\text { en vías de convertirse en sociedades del } \\
\text { dopaje. }\end{array}$ \\
\hline Libertad & $\begin{array}{l}\text { En Castells el concepto libertad adquiere } \\
\text { una connotación positiva, pues se señala } \\
\text { que fue precisamente el sentido de la } \\
\text { libertad la que permitió el desarrollo } \\
\text { de una tecnología (digital) que a su vez } \\
\text { fomenta ese sentido de la libertad y la } \\
\text { participación. }\end{array}$ & $\begin{array}{l}\text { El concepto en Byun-Chul Han adquiere } \\
\text { un sentido negativo no por su ausencia, } \\
\text { sino porque ese sentido de la libertad } \\
\text { en las sociedades contemporáneas en } \\
\text { realidad no solamente es ficticio, sino que } \\
\text { conlleva su contrario es decir sumisión a } \\
\text { los dictados del poder neoliberal. }\end{array}$ \\
\hline
\end{tabular}

FUENTE: ELABORACIÓN PROPIA. 
COMUNICACIÓN

PERSPECTIVA DE MANUEL CASTELLS

Castells define a la comunicación como (2006, p. 88) interactiva caracterizada por la capacidad para enviar mensajes de muchos a muchos, en tiempo real, o en un momento concreto, y con la posibilidad de usar la comunicación punto-a-punto, estando el alcance de su difusión en función de las características de la práctica comunicativa perseguida.
PERSPECTIVA DE MARSHALL MC.LUHAN

Marshall McLuhan entiende a la comunicación en relación con la tecnología y establece que todos los medios son prolongaciones de alguna facultad humana, psíquica o física. Así, señala McLuhan (1993, p. 74), que contemplar, utilizar o percibir cualquier prolongación de nosotros mismos en forma técnica implica adoptarla forzosamente.

FUENTE: ELABORACIÓN PROPIA.

Para Manuel Castells, el fenómeno comunicativo es pieza fundamental que da sentido al concepto de sociedad red. De hecho, el vínculo comunicación y microelectrónica fue el factor que le suministró un sentido particular al modelo de sociedad red que organiza a las sociedades contemporáneas. Así, cuando Castells se refiere a la microelectrónica, en realidad se está refiriendo a todos aquellos avances tecnológicos que han permitido el desarrollo de un sistema de redes electrónicas que posibilitaron el nacimiento de Internet.

Por ello, es imposible entender el concepto de Castells al margen de la tecnología. Así, el autor señala que (2009, p. 87) el proceso de comunicación se define por la tecnología de la comunicación, las características de los emisores y los receptores de la información, sus códigos culturales de referencia, sus protocolos de comunicación y el alcance del proceso.

Así, cuando Castells se refiere al fenómeno comunicativo, pasa del modelo de comunicación social planteada por la Mass Communication Research y del modelo de comunicación circular propio de la comunicación interpersonal (rica en niveles y posibilidades de retroalimentación directa). En realidad, el modelo de Castells es un proceso multinivel (que incluye lo interpersonal, lo grupal y lo global), reticular, participativa, con formas de retroalimentación diversas y que se adapta a las necesidades de los diferentes públicos.

De la misma manera, Castells llama al modelo de comunicación que propone autocomunicación de masas, porque (2009, p. 88) potencialmente puede llegar a una audiencia global, como cuando se cuelga un video en YouTube, un blog con enlaces RSS a una serie de webs o un mensaje a una lista enorme de direcciones de correo electrónico. A todo esto, habría que agregarle la rapidez y la relativa facilidad que representa llevar a cabo todas estas acciones, las cuales en otras épocas estaban fuera del alcance de las manos de los ciudadanos. 
Al mismo tiempo, complementa que el modelo que plantea es autocomunicación porque (2009, p. 88) uno mismo genera el mensaje, define los posibles receptores y selecciona los mensajes concretos o los contenidos de la web y de las redes de comunicación electrónica que quiere recuperar. De tal manera, la autocomunicación no implica que se generen mensajes para un consumo estrictamente personal, sino por el contrario se refiere a los roles que estaría jugando el ciudadano de la sociedad red. En tal sentido estos ciudadanos (o nodos) estarían en condiciones no solo de ser consumidores y productores de mensajes, sino de seleccionar, adaptar, modificar, extender o contraer tales mensajes y los procesos de interacción a sus necesidades y capacidades.

Es decir, la autocomunicación de masas propuesta por Manuel Castells se vincularía de acuerdo con el propio autor, con el concepto convergencia de Henry Jenkins, ya que en este modelo confluyen formas, niveles y tecnologías de comunicación e información, pero la verdadera significación, agrega Castells (2009, p. 88), se produce dentro del cerebro de los consumidores individuales y a través de su interacción social con los demás. Y tal interacción, habría que agregar, se estaría generando de manera inmediata, virtual y con un alcance global potencializado por las ventajas de la tecnología de la microelectrónica.

Por su parte, Marshall McLuhan establece una relación directa entre la comunicación y la tecnología. De hecho, siguiendo a Harold Innis, McLuhan establece que cada revolución tecnológica que impacta en las maneras de comunicar un mensaje trae consigo pequeñas o grandes revoluciones en el ámbito económico y político.

Así, para entender a McLuhan habría que comenzar por señalar que se debe establecer una diferenciación entre las reales capacidades del hombre (otorgadas por la naturaleza) y la manera en la que la tecnología no solo impacta en esas capacidades, sino que las prolonga, las extiende. En tal sentido señala que (1962, p. I3) el hombre, ese animal que construye instrumentos, sea el lenguaje, la escritura o la radio, se ha dedicado desde hace mucho tiempo a ampliar uno u otro de sus órganos sensoriales, pero lo ha hecho de tal modo que todos los restantes sentidos o facultades han sufrido extorsión.

Dicho de otra manera, la tecnología desde la perspectiva de McLuhan es una prolongación de las capacidades humanas a las cuales, para bien o para mal, altera. Agrega McLuhan que contemplar, utilizar o percibir cualquier prolongación de nosotros mismos (1993, p. 74) en forma técnica implica adoptarla forzosamente. Escuchar radio o leer la página impresa equivale a que aceptemos dentro de nuestro sistema personal estas prolongaciones de nosotros mismos y que suframos el "cierre" o desplazamiento de la percepción que le sigue automáticamente.

Es decir, en el proceso de relación entre sujeto-objeto la mediación de la tecnología en muchas ocasiones estaría impactando los significados que habrán de construirse. Así, 
la imagen cinematográfica gigantesca, vívida y colorida generaban en el espectador una curiosa sensación en donde se ganaba en percepción, pero se restaba en capacidad imaginativa que podría generar la misma historia, por así decirlo narrada en la radio.

Así, tal como Castells establece que los avances de la tecnología microelectrónica constituyeron la columna vertebral de los cambios en las sociedades contemporáneas, para McLuhan el gran detonante fue la luz eléctrica. De tal manera señala que (1969, p. 5) la tecnología eléctrica está remodelando y reestructurando los patrones de la interdependencia social y cada uno de los aspectos de nuestra vida.

Por tal efecto, habría que entender que en la dicotomía medio-mensaje que establece McLuhan, ni el medio es el mensaje, ni viceversa. En realidad, para McLuhan el medio es pura tecnología (prolongación de las extremidades humanas). Representa la parte externa y tangible del proceso de comunicación que apunta hacia lo interno, lo intangible, lo espiritual del ser humano. El dilema consiste en que, en esas condiciones, la tecnología llega a ser tan importante que el contenido queda relegado a un segundo plano ante una red generadora de emociones y sensaciones seductoras. La comunicación rápida, acota McLuhan (2002, p. 26), sirve para limpiar y olvidar más que para aprender y acumular conocimientos.

En tal caso, para McLuhan (1989, p. 25) las tecnologías, al igual que las palabras, son metáforas. De este modo, comprometen la transformación del usuario en tanto que establecen nuevas relaciones entre éste y sus medios. Relaciones que son emociones, vivencias, en donde no es tan importante lo que se vive, sino el modo en el que se vive. Tal vez, esta sea una de las razones del éxito de las redes sociodigitales, en donde fluyen grandes cantidades de información, mucha de ella sin profundidad, pero capaces de despertar las emociones más diversas.

\section{COINCIDENCIAS/DIFERENCIAS MCLUHAN Y CASTELLS}

\begin{tabular}{|l|l|}
\hline \multicolumn{1}{|c|}{ COINCIDENCIAS } & \multicolumn{1}{c|}{ DIFERENCIAS } \\
\hline $\begin{array}{l}\text { Ambos coinciden en que la comunicación } \\
\text { es la columna vertebral de las sociedades } \\
\text { contemporáneas. }\end{array}$ & $\begin{array}{l}\text { El concepto de comunicación en Castells apunta } \\
\text { hacia la convergencia de tecnologías, niveles, } \\
\text { intereses, etcétera. }\end{array}$ \\
\hline $\begin{array}{l}\text { Ambos establecen una perspectiva de comunicación } \\
\text { ligada a la tecnología. }\end{array}$ & $\begin{array}{l}\text { En McLuhan la comunicación está centrada en } \\
\text { gran medida en el medio el cual proporciona } \\
\text { características precisas a los mensajes. }\end{array}$ \\
\hline
\end{tabular}




\section{COINCIDENCIAS}

Ambos consideran que el surgimiento de determinados avances tecnológicos (en Castells la microelectrónica y en McLuhan la electricidad) representaron revoluciones tan profundas que impactaron en el surgimiento de nuevos paradigmas sociales.

Ambos consideran a la tecnología como detonante de las capacidades de ser humano.

Ambos utilizan el adjetivo potenciación para referirse al mejoramiento de las capacidades (humanas, de las redes, de los medios de comunicación, etcétera).

\section{DIFERENCIAS}

Castells llama a su proceso autocomunicación dado el alcance global que adquieren los mensajes y los diversos roles que juega el ciudadano en el proceso de comunicación.
Para McLuhan, el ser humano ve condicionada manera en la que se vincula con los mensajes por el tipo de medio que utiliza.

Para Manuel Castells, el individuo está en condiciones de consumir y generar mensajes y al mismo tiempo de seleccionar y adaptar los mismos generando significaciones particulares a los menajes de acuerdo con su perfil, a sus preferencias y a sus necesidades.

Los significados para McLuhan están relacionados con las características de los medios más que con las características de los receptores.

Para Marshall McLuhan no es tan importante lo que dice el mensaje, sino cómo se entra en contacto con él y que sensaciones llega a generar.

FUENTE: ELABORACIÓN PROPIA.

\section{VALORACIÓN DE LOS CONCEPTOS}

\begin{tabular}{|l|l|l|}
\hline \multicolumn{1}{|c|}{ CONCEPTO } & \multicolumn{1}{|c|}{ MANUEL CASTELLS } & \multicolumn{1}{c|}{ MARSHALL MCLUHAN } \\
\hline Comunicación & $\begin{array}{l}\text { El concepto comunicación en Castells } \\
\text { es positivo, pues la autocomunicación } \\
\text { de masas permite la vinculación de } \\
\text { las personas a nivel global de manera } \\
\text { relativamente sencilla. A su vez dicho } \\
\text { individuo es capaz de involucrarse en } \\
\text { el proceso de comunicación no solo } \\
\text { consumiendo y generando mensajes, sino } \\
\text { que está en capacidad de elegir yadaptar } \\
\text { dichos contenidos a sus necesidades. }\end{array}$ & $\begin{array}{l}\text { Adquiere un sentido negativo puesto } \\
\text { que por una parte la comunicación se } \\
\text { ve potenciada por la tecnología, pero } \\
\text { esto a la larga resulta contradictorio } \\
\text { pues la importancia del mensaje es } \\
\text { desplazada por la eficiencia de los } \\
\text { medios y por su capacidad de generar } \\
\text { diversas sensaciones en la audiencia. }\end{array}$ \\
\hline
\end{tabular}




\begin{tabular}{|l|l|l|}
\hline \multicolumn{1}{|c|}{ CONCEPTO } & \multicolumn{1}{|c|}{ MANUEL CASTELLS } & \multicolumn{1}{c|}{ MARSHALL MCLUHAN } \\
\hline Tecnología & $\begin{array}{l}\text { La connotación de la tecnología en Castells } \\
\text { es positiva, ya que fueron precisamente los } \\
\text { avances en la tecnología microelectrónica } \\
\text { los que permitieron el desarrollo de una } \\
\text { cultura digital altamente participativa, } \\
\text { libre y global. }\end{array}$ & $\begin{array}{l}\text { El sentido que adquiere el concepto de } \\
\text { tecnología en McLuhan es neutro, ya } \\
\text { que conlleva un sentido positivo y uno } \\
\text { negativo. Es decir, para McLuhan los } \\
\text { medios (la tecnología) es prolongación } \\
\text { de las extremidades del hombre. } \\
\text { En tal prolongación algunas de las } \\
\text { capacidades del ser humano se } \\
\text { ven potenciadas (adquieren mayor } \\
\text { velocidad, fuerza o capacidad de } \\
\text { percepción)y otras se ven disminuidas } \\
\text { (como la memoria, la imaginación, } \\
\text { etcétera). }\end{array}$ \\
\hline
\end{tabular}

FUENTE: ELABORACIÓN PROPIA.

\section{CONCLUSIONES}

Reflexionar sobre las implicaciones del concepto sociedad red desde la perspectiva planteada por Manuel Castells y compararla con las ideas desarrolladas por Han y McLuhan, resultó no solamente aleccionador, sino una tarea necesaria, y en extremo compleja, pues involucra afinar una mirada analítica y crítica sobre las nuevas tecnologías digitales, además una perspectiva amplia que trascienda el maniqueísmo tecnológico y pueda ser capaz de aceptar que la sociedad red es una realidad que de algún modo hoy en día involucra al tejido social entero, aun a aquellos que están marginados del acceso a las redes sociodigitales.

De igual manera, a partir del presente estudio pudimos descubrir que en el análisis de la sociedad red, junto a los conceptos sociedad y comunicación, aparecen muy vinculados los conceptos de libertad y tecnología.

Así, los conceptos sociedad, comunicación, libertad y tecnología de alguna manera se combinan para configurar lo que Manuel Castells llama sociedad red. Lo relevante del caso es que a partir del análisis realizado podemos señalar que tales conceptos en Castells encarnan una visión bastante positiva u optimista del entorno social contemporáneo. Es decir, a pesar de que Castells es enfático al reconocer que grandes grupos de personas son marginadas de las bondades de la conectividad, en términos generales los conceptos vinculados a la sociedad red se relacionan al sentido de libertad, progreso, rapidez y senti- 
do de pertenencia a una comunidad global participativa a partir de redes (tecnológicas y humanas) que conectan al hombre con el mundo.

Por el contario, Byung-Chul Han desarrolla una perspectiva crítica sobre los conceptos sociedad y libertad. Así, Han utiliza adjetivos que connotan negatividad para referirse a las sociedades contemporáneas. Algunas de estas palabras son: cansancio, rendimiento y fundida. Incluso llega a afirmar que se trata de una colectividad en vías de convertirse en sociedades del dopaje.

De igual manera para Han, el sentido que adquiere el concepto libertad en las sociedades contemporáneas es de alguna manera negativo porque tal libertad más que ser real es mera sensación que no llega a trascender. Se trata de una peculiar sensación de libertad, alentada en muchas ocasiones por factores como la lejanía entre los interlocutores en las redes sociodigitales, la inmediatez y el poder que otorga el anonimato (total y/o parcial) que impide la rendición de cuentas, la deliberación y una comunicación plena.

Así entendida, esta sensación de libertad en red para Han, en realidad es una especie de continuación de la explotación generada por el propio sistema, tendiente inclusive a incidir más allá de la jornada laboral, ubicándose en el tiempo libre de las personas, e impactando de manera cálida, gozosa y, por lo mismo adictiva, pero inservible en el sentido práctico.

De igual manera, a partir del análisis realizado pudimos encontrar en Castells la palabra potenciada como un adjetivo que califica a las organizaciones en red contemporáneas y a los procesos de comunicación. Tal potenciación estaría vinculada al desarrollo de las microtecnologías, es decir, por la tecnología digital. Así, para Castells el detonante que permitió el paso del paradigma industrial al digital lo constituyeron justamente los avances tecnológicos en el campo de la microtecnología.

Algo similar señala McLuhan, quien establece que en el pasado siglo tal factor de cambio lo significó la luz eléctrica, la cual denota para McLuhan energía pura, chispa que estuvo en capacidad de generar una explosión social caracterizada sobre todo por el poder que adquirieron los medios masivos y la comunicación satelital en el siglo xx.

No obstante, de acuerdo con el análisis realizado, la perspectiva de McLuhan sobre la tecnología es neutro, ya que su utilización genera consecuencias tanto positivas como negativas. Es decir, por un lado, McLuhan también utiliza la palabra potenciación para referirse a la manera en la que la tecnología impacta en las extremidades o capacidades del hombre potencializándolas. Pero tales efectos magnificadores de las capacidades humanas traen consigo la paradoja de anular otros sentidos o capacidades. Como en la metáfora de Narciso frente al espejo de agua, a la cual hace referencia el propio McLuhan, que enamora y mata al mismo tiempo. 
En ese mismo tenor, la perspectiva que McLuhan desarrolla sobre la comunicación, de alguna manera denota un sentido negativo, ya que la comunicación y los medios potenciados por la tecnología deslumbran al espectador con lo cual se produce que sea desplazada la importancia del mensaje por las sensaciones que genera el contacto con los medios. Así, el qué (contenido) queda desplazado por el cómo (los medios y las sensaciones que genera), lo cual impacta no de manera positiva en los significados construidos por el hombre.

Así, este nuevo modelo de sociedad se nos presenta amable, optimista y desbordado de elementos seductores. La ciencia y la tecnología por fin al servicio del hombre; todos conectados, iluminados por luz eléctrica que, como en McLuhan, significa información y energía pura: todos portando la evolución tecnológica en nuestras espaldas, como una segunda piel. Las capacidades humanas magnificadas con la ayuda de la tecnología. Nuestras extremidades nunca fueron más eficientes de lo que son ahora. Y el cerebro, metaforizado por los circuitos eléctricos de las computadoras, poseedoras de una memoria suficiente para contener el conocimiento del mundo. No obstante, señalaría el propio McLuhan cuando una extremidad es potencializada, alguna otra queda disminuida.

Así pues, por una parte, es claro que el modelo de sociedad red es aún muy reciente (pero necesita ser cuestionado), y la ciudadanía se encuentra en el tránsito de un mundo que gira en torno a las dinámicas de un contexto moderno e industrial, pero que se mueve a uno global y ampliamente interconectado, debido, entre otras cosas, a la tecnología digital.

Por todo esto, resulta importante entender a cabalidad que, como lo señala Castells, el concepto sociedad red involucra una parte tecnológica y otra sociocultural. Esto permitirá poner a Internet en sus justas dimensiones y considerar que, en torno a lo que sucede en esta plataforma, solo el espacio es virtual, ya que en las demás partes del proceso de interacción y comunicación (en las formas de participación, en las emociones que se generan, en los usos que se le asignan, etcétera) está involucrada, en mayor o menor medida la experiencia del ser humano.

De tal manera, a partir del análisis del concepto sociedad red, partiendo de la visión que se tiene en tal paradigma de los conceptos sociedad y comunicación podemos corroborar que, en efecto, hoy más que nunca las sociedades contemporáneas desarrollan sus rutinas en la vida cotidiana, en su entorno laboral, escolar, comercial, etcétera, haciendo uso de las tecnologías de información y comunicación.

Así, la dicotomía sociedad-comunicación involucra en medio a una red de relaciones y contactos que permiten formas de comunicación potenciadas por tales tecnologías. Dicho de otra manera, tal parece que en ningún momento de la historia el hombre había tenido tan alcance de sus manos avances tecnológicos capaces de conectarlo globalmente. Lo cual, a partir de lo aquí planteado, en Castells significaría progreso generado por los avances de la 
microelectónica, pero para Han y McLuhan denotarían una paradoja altamente peligrosa para el conglomerado social.

\section{REFERENCIAS}

Alva, G. (200I). Métodos de análisis de los mensajes. Bogotá: Pontificia Universidad Javeriana.

Asociación Mexicana de Internet. (2019). $15^{\circ}$ Estudio Sobre los Hábitos de los Usuarios de Internet en México 20I9. México: AMIPCI.

Castells, M. (200I). La galaxia Internet. Madrid: Plaza \& Janés.

Castells, M. (2006). La sociedad red: una visión global. Madrid: Alianza Editorial.

Castells, M. 2009. Comunicación y poder. Madrid: Alianza Editorial.

Han, B-C. (2OI2). La sociedad del cansancio. Barcelona: Herder.

Han, B-C. (2014). Psicopolítica. Barcelona: Herder.

Hauben, R. (1993). The International and Scientific Origins of the Internet and the Emergence of the Netizens. Ronda Hauben. Recuperado de http://www.ais.org/ jrh/acn/acn15-2.articles/ rhauben.pdf

McLuhan, M. (1969). El medio es el mensaje. Barcelona: Paidós.

McLuhan, M. y Powers, B. R. (1989.) La aldea global. Barcelona: Gedisa.

Terceiro B. J. y Matías, G. (200I). Digitalismo. El nuevo borizonte sociocultural. Madrid: Taurus Digital. 